\title{
Rechte und Pflichten von niedergelassenen Radiologen im Insolvenzverfahren
}

Die Insolvenz von Vertragsärzten spielt quantitativ zwar nach wie vor keine bedeutende Rolle. In der Radiologie können jedoch gerade größere Praxisstrukturen, wie überörtliche Gemeinschaftspraxen und MVZ, durch unwirtschaftliches Verhalten der Gesellschafter oder auch durch unvorhergesehene Veränderungen in der vertragsärztlichen Honorarsituation in eine wirtschaftliche Schieflage geraten. Eine dünne Kapitaldecke führt dann häufig zu einer Überschuldung und einer anschließenden Zahlungsunfähigkeit. Dies zeigt das Beispiel einer Hamburger Radiologiegesellschaft, die mit ihren insgesamt 7 MVZ-Gesellschaften Ende des Jahres 2012 in die Insolvenz geraten ist.

Die Durchführung eines Insolvenzverfahrens in einer Arztpraxis ist auch für erfahrene Insolvenzverwalter in rechtlicher und tatsächlicher Hinsicht anspruchsvoll, da verschiedene Rechtsgebiete zu beachten und aufeinander abzustimmen sind. Die hat Auswirkungen zum einen auf die klassischen Beteiligten des Insolvenzverfahrens (Gläubiger, Schuldner und Insolvenzverwalter), aber auch auf die Patienten und die Kassenärztlichen Vereinigungen (KV). Dabei sind die Rechte und Pflichten aus der Insolvenzordnung mit den Besonderheiten des Vertragsarztrechts gegen- und miteinander abzugrenzen. Dies gilt insbesondere für den Bereich der ver- tragsärztlichen Leistungsabrechnung gegenüber den Kassenärztlichen Vereinigungen. Oftmals hat der insolvente Vertragsarzt nur noch ein geringes Interesse an der Durchführung der Abrechnung gegenüber der KV, da die Vergütung überwiegend oder vollständig an seine Gläubiger weitergeleitet wird. Der nachfolgende Beitrag beschäftigt sich mit den Rechten und Pflichten von Insolvenzverwaltern und Vertragsärzten im Rahmen des Insolvenzverfahrens, aber auch mit den entsprechenden Gefahren. Er setzt sich dabei auch mit den Mitwirkungsrechten und -pflichten der betroffenen Vertragsärzte und den entsprechenden Befugnissen der Insolvenzverwalter auseinander.

\section{Einleitung: Überblick über die Anzahl der Insolvenzen von Vertragsärzten $\nabla$}

Bei den Insolvenzen von Vertragsärzten ${ }^{1}$ handelt es sich um einen Themenbereich, der eher ein Schattendasein führt. Eine aktuelle Statistik ist insoweit nicht bekannt. Die wohl aktuellsten Zahlen stammen von der Wirtschaftsauskunftsdatei Creditreform aus den Jahren 2005 bzw. 2006. ${ }^{2}$ Im Jahr 2005 betrug die Zahl der Insolvenzen von Arzt- und Zahnarztpraxen insgesamt 240 bei insgesamt ca. 175.000 niedergelassenen Ärzten und
Zahnärzten; ${ }^{3}$ dies entspricht einer Insolvenzquote von ca. 0,15 Prozentpunkten. Die Zahl der niedergelassenen Ärzte und Zahnärzte ist in den folgenden Jahren relativ konstant geblieben; im Jahr 2010 betrug diese Zahl ca. 176000. ${ }^{4}$ Daraus könnte man ableiten, dass sich auch die Zahl der Insolvenzen nicht wesentlich verändert haben dürfte. Zum Vergleich dazu branchenübergreifend die Zahl der Insolvenzen: Im Jahr 2005 betrug die Anzahl der Insolvenzen insgesamt 36.843 bei insgesamt 3036758 umsatzsteuerpflichtigen Unternehmen ${ }^{5}$ dies entspricht einer Quote von ca. 1,21 Prozentpunkten. Im Jahre 2010 gab es 3165286 umsatzsteuerpflichtige Unternehmen und 31998 Insolvenzen, ${ }^{6}$ was einer Quote von 1,16 Prozentpunkten entspricht. ${ }^{7}$ Auf Ärzte und Zahnärzte bezogen ist insgesamt festzustellen, dass Vertragsärzte und Vertragszahnärzte wohl generell einem erheblich geringeren Insolvenzrisiko ausgesetzt sind, als dies im Durchschnitt der Unternehmen in Deutschland der Fall ist. 
Vor diesem Hintergrund soll nunmehr erörtert werden, wie sich die Situation darstellt, wenn über das Vermögen eines Arztes ein Insolvenzverfahren eröffnet worden ist, dieser aber noch Forderungen aus vertragsärztlich erbrachten Leistungen gegen seine Kassenärztliche Vereinigung hat, seinen Mitwirkungspflichten insoweit aber nicht nachkommt. Denn Folge der unzureichenden Mitwirkung ist zum einen, dass die Ansprüche der Gläubiger beeinträchtigt werden können. Weitere Folge ist, dass mit Eröffnung des Insolvenzverfahrens die Verwaltungs- und Verfügungsbefugnis über das Vermögen des insolventen Vertragsarztes auf den Insolvenzverwalter übergeht, der insoweit eine besondere Pflichtenstellung einnimmt. ${ }^{8}$ Wenn es der Insolvenzverwalter daher unterlässt, Forderungen des insolventen Vertragsarztes geltend zu machen, macht er sich selber möglicherweise strafbar. ${ }^{9}$

\section{Rechtliche Grundlagen \\ $\nabla$}

Das Vertragsarztrecht ist im Wesentlichen im SGB V geregelt; ergänzende Regelungen finden sich in Verordnungen und Verträgen. Rechtsgrundlage für Honorarverteilungsbestimmungen ist $\S 87 \mathrm{~b}$ SGB V. Danach verteilen die Kassenärztlichen Vereinigungen die Gesamtvergütungen an die Vertragsärzte getrennt für die Bereiche hausärztlichen und fachärztlichen Versorgung (§73 SGB V). Seit Inkrafttreten des GKV-Versorgungsstrukturgesetzes zum 1.1.2012 (GKV-VStG) beschließen die Vertreterversammlungen der KVen die Honorarverteilungsregeln per Honorarverteilungsmaßstab (HVM); ${ }^{10}$ als Beispiel für die weiteren Ausführungen dient der Honorarverteilungsmaßstab der KV Nordrhein vom 15.5.2012, der sich seit dem 1.7.2012 in Kraft befindet. ${ }^{11}$

Auf der anderen Seite befasst sich das Insolvenzrecht als Teil des Zivilrechts in verfahrens- und materiellrechtlicher Hinsicht mit den Rechten von Gläubigern bei der Insolvenz von Schuldnern. Eröffnungsgründe für ein Insolvenzverfahren sind nach $\S \S 16$ ff. Insolvenzordnung (InsO) Zahlungsunfähigkeit, drohende Zahlungsunfähigkeit und Überschuldung. Das Ziel eines Insolvenzverfahrens besteht darin, entweder die Zahlungsfähigkeit wieder herzustellen oder das Unternehmen durch geordnete Abwicklung aufzulösen. ${ }^{12}$ Mit Eröffnung des Insolvenzverfahrens geht das Recht des Schuld- ners, das zur Insolvenzmasse gehörende Vermögen zu verwalten und über es zu verfügen, auf den Insolvenzverwalter über. Die rechtlichen Grundlagen des Insolvenzverfahrens sind in der InsO festgelegt.

Beide Rechtsbereiche, d.h. das SGB V und die InsO stehen von ihrem Gesetzesrang auf einer Ebene. Es ergibt sich keine Anwendbarkeit von Kollisionsregeln. In Anlehnung an das Prinzip der sog. praktischen Konkordanz folgt daraus für die eingangs benannte Problematik, dass die jeweils geschützten Rechtsbereiche in der Problemlösung einander so zugeordnet werden müssen, dass jedes von ihnen Wirklichkeit gewinnt. Insbesondere müssen beiden Bereichen Grenzen gesetzt werden, damit beide zu optimaler Wirksamkeit gelangen können. ${ }^{13}$

\section{Fortsetzung der Vertragsarzt- praxis nach Insolvenzeröffnung $\nabla$}

Bei einer Insolvenz seiner Vertragsarztpraxis kann der Vertragsarzt diese mit fachlicher Unabhängigkeit fortführen. Dies ergibt sind unmittelbar aus §95 Abs. 6 S. 1 SGB V. Danach ist die Zulassung zu entziehen, „wenn ihre Voraussetzungen (Anm. d. Verf.: für eine Zulassung) nicht oder nicht mehr vorliegen, der Vertragsarzt die vertragsärztliche Tätigkeit nicht aufnimmt oder nicht mehr ausübt oder seine vertragsärztlichen Pflichten gröblich verletzt.“

Eine Insolvenz stellt jedoch in der Regel keine „gröbliche vertragsärztliche Pflichtverletzung" dar. Nach der Rechtsprechung des Bundessozialgerichts (BSG) ist eine Pflichtverletzung dann gröblich, wenn sie so schwer wiegt, dass ihretwegen die Entziehung zur Sicherung der vertragsärztlichen Versorgung notwendig ist. Davon ist dann auszugehen, wenn durch sie das Vertrauen der vertragsärztlichen Institutionen in die ordnungsgemäße Behandlung der Versicherten und in die Rechtmäßigkeit der Abrechnungen durch den Vertragsarzt so gestört ist, dass ihnen eine weitere Zusammenarbeit mit dem Vertragsarzt nicht mehr zugemutet werden kann. Nicht erforderlich ist, dass den Vertragsarzt ein Verschulden trifft; auch unverschuldete Pflichtverletzungen können zur Zulassungsentziehung führen. ${ }^{14}$ Die Zulassungsentziehung darf unter Berücksichtigung des Verhältnismäßigkeitsgrundsatzes nur ausgesprochen werden, wenn sie das einzige Mittel zur Sicherung und zum Schutz der vertragsärztlichen Versorgung ist (ultima ratio). ${ }^{15}$ Da vor diesem Hintergrund in einer Insolvenz an sich keine gröbliche Pflichtverletzung im Sinne des $§ 95$ Abs. 6 S. 1 SGB V zu sehen ist, hat diese in der Regel keine Auswirkungen auf die vertragsärztliche Zulassung. ${ }^{16}$

Es kommt hinzu, dass die vertragsärztliche Zulassung nach der Rechtsprechung des BSG nicht in die Insolvenzmasse fällt. Die Zulassung als Vertragsarzt stellt sich als Zuerkennung einer öffentlich-rechtlichen Berechtigung durch Stellen staatlicher Verwaltung, nämlich der Zulassungsund Berufungsausschüsse ( $\S 996,97$ SGB V), dar. Mit ihr wird dem Berechtigten die Befugnis übertragen, im System der gesetzlichen Krankenversicherung die Versicherten gesetzlicher Krankenkassen mit Wirkung für diese zu behandeln. Die $\mathrm{Zu}-$ lassung ist daher untrennbar mit der Person des Berechtigten verbunden. Als solchermaßen ausgestaltete öffentlichrechtliche Berechtigung ist die Zulassung als Vertragsarzt ebensowenig übertragbar oder pfändbar wie etwa der Status als Rechtsanwalt. ${ }^{17}$ Als öffentlich-rechtliche Berechtigung kann die Zulassung bei Vermögensverfall des Vertragsarztes nicht in die Konkursmasse fallen mit der Folge, dass der Konkursverwalter über sie verfügen und sie verwerten könnte. ${ }^{18}$ Sämtliche Anträge in Bezug auf die Zulassung, wie z.B. der Verzicht, die Durchführung des Ausschreibungsverfahrens nach $§ 103$ Abs. 4 SGB V oder die Verlegung der Zulassung können daher nur durch den Vertragsarzt persönlich und nicht durch den Insolvenzverwalter gegenüber den Zulassungsgremien beantragt werden.

Ein weiterer Aspekt ergibt sich aus $\S 80$ Abs. 1 InsO. Danach geht das Recht des Schuldners, das zur Insolvenzmasse gehörende Vermögen zu verwalten und über es zu verfügen, mit Eröffnung des Insolvenzverfahrens auf den Insolvenzverwalter über. Man könnte daher zunächst davon ausgehen, dass der Insolvenzverwalter die Vertragsarztpraxis umfassend betreibt. Dass dem aber nicht so ist, versteht sich grundsätzlich von selbst. Denn nur der Vertragsarzt verfügt über das fachliche Wissen; eine Fortführung durch einen Insolvenzverwalter ist daher rechtlich nicht möglich ist, jedenfalls soweit er nicht nach $\S 2$ Abs. 1 BÄO auch approbierter Arzt ist. ${ }^{19}$ Zudem ist zu bedenken, dass oftmals zwischen Arzt und seinen Patienten ein be- 
sonderes Vertrauensverhältnis besteht. Folge ist, dass der Insolvenzverwalter insoweit nicht an die Stelle des Vertragsarztes als Schuldner treten darf und kann.

Soweit der Fortsetzung der Vertragsarztpraxis auch keine weiteren Gründe entgegenstehen (z.B. Berufsordnung, Approbation nach $\mathrm{BÄO})^{20}$ darf der Vertragsarzt seine Praxis auch nach Insolvenzeröffnung weiter betreiben. Andererseits verliert der Vertragsarzt als Insolvenzschuldner die Verfügungsbefugnis über sein Vermögen. Dies gilt nur dann nicht, wenn es sich um eine höchstpersönliche Angelegenheit des Schuldners, wie z.B. Erbschafts-, Scheidungs- oder Strafverfahren, oder um sonstige Rechtsstreitigkeiten über insolvenzfreies Vermögen handelt. Einkünfte aus einer Tätigkeit als Vertragsarzt sind jedoch keine höchstpersönlichen Rechte, sondern gehören in vollem Umfang zur Insolvenzmasse und sind an den Insolvenzverwalter auszukehren. ${ }^{21}$

\section{Vergütungsanspruch gegen die Kassenärztliche Vereinigung \\ $\nabla$}

Wesentlich für die vertragsärztliche Vergütung ist der Honorarverteilungsmaßstab (HVM), im Bereich der KVNO der HVM der KV Nordrhein vom 15.5.2012, in Kraft seit dem 1.7.2012. ${ }^{22}$ Im Rahmen eines Insolvenzverfahrens ist jedoch fraglich, wer die Rechnungslegung vornimmt. Infrage kommen insoweit der Vertragsarzt und / oder der Insolvenzverwalter.

\section{Abrechnung, Rechnungslegung}

Nach $§ 1$ Abs. 1 HVM KVNO sind grundsätzlich alle zur ärztlichen Behandlung und Betreuung gehörenden Leistungen im Rahmen der vertragsärztlichen Versorgung gemäß §73 Abs. 2 SGB V abrechnungsfähig, mit Ausnahme der zahnärztlichen und kieferorthopädischen Behandlung und der Versorgung mit Zahnersatz einschließlich Zahnkronen und Suprakonstruktionen nach Maßgabe der Nrn. 2 und 2a des $\S 73$ Abs. 2 SGB V. Die Leistungen müssen nach den für die Durchführung maßgeblichen Bestimmungen des SGB V und der darauf gründenden Regelungen erbracht worden sein. Nicht abrechnungsfähig sind u.a. Untersuchungen und Behandlungen aus betriebsärztlicher Tätigkeit, die Selbstbehandlung sowie die Behandlung von Familienangehörigen oder Leistungen im Auftrag des medizinischen Dienstes.
Nach $\S 1$ Abs. 2 HVM KVNO ist die Abrechnung vertragsärztlicher Leistungen quartalsweise vorzunehmen. Die Rechnungslegung ist persönlich - ohne die Einschaltung von Dritten, insbesondere sog. Verrechnungsstellen, soweit nicht ausdrücklich gesetzlich oder vertraglich vorgesehen - vorzunehmen. Die aufgrund unzulässiger Datenverarbeitung erstellte $\mathrm{Ab}$ rechnung darf nicht verwendet werden und wird zurückgewiesen.

Im Falle einer sich im Insolvenzverfahren befindlichen Vertragsarztpraxis könnte für die Rechnungslegung aus dem Wortlaut des $\S 80$ Abs. 1 InsO folgen, dass diese ausschließlich vom Insolvenzverwalter als zentrale Figur des Insolvenzverfahrens und nicht vom Vertragsarzt vorgenommen werden darf; ${ }^{23}$ denn dem Insolvenzverwalter wird grundsätzlich ohne Einschränkungen die Verwaltungs- und Verfügungsbefugnis über das Vermögen des Schuldners übertragen. ${ }^{24}$ Hierfür spricht auch, dass nach $\S 56$ Abs. 1 InsO das Insolvenzgericht eine für den jeweiligen Einzelfall geeignete, insbesondere geschäftskundige Person als Insolvenzverwalter zu bestellen hat. Auch heißt es in $\S 148$ Abs. 1 InsO, dass der Insolvenzverwalter nach Eröffnung des Insolvenzverfahrens das gesamte zur Insolvenzmasse gehörende Vermögen sofort in Besitz und Verwaltung zu nehmen hat. Allein vor diesen Hintergründen wäre der Insolvenzverwalter grundsätzlich in der Lage, die Abrechnung anhand der vorhandenen Dokumentation des Vertragsarztes zu erstellen.

Gegen die Annahme einer Rechnungslegung durch den Insolvenzverwalter bestehen jedoch erhebliche Bedenken. Denn wenn der Insolvenzverwalter die ärztliche Dokumentation sichtet, steht damit gleichzeitig ein Verstoß gegen die ärztliche Schweigepflicht im Raum. Unter den Begriff Dokumentation ist eine Zusammenstellung von Beweisstücken zu verstehen, die der Arzt oder andere Hilfspersonen des Arztes im Zuge der Behandlung entweder selbst erzeugt oder vom Patienten oder einem Dritten erhalten hat. Eine Dokumentation umfasst daher u.a. folgende Unterlagen: Ärztliche Niederschriften, Krankenkarteikarten, OP-Berichte, Röntgenaufnahmen, MRT-Bilder, Videoaufnahmen einer OP oder Lichtbilder. ${ }^{25}$ Hieraus lässt sich ein Anspruch des Patienten auf Einhaltung der Schweigepflicht herleiten, dessen Grundlage sich aus dem allgemeinen Persönlichkeitsrecht nach Art. 1 Abs. 1, Art. 2 Abs. 1 GG ergibt. Der dahinter stehende Zweck besteht zum einen im Schutz der Geheimsphäre des einzelnen Patienten und zum anderen in dem Interesse der Allgemeinheit, dass das Vertrauensverhältnis zwischen Ärzten und Patienten nicht beeinträchtigt werden darf. $^{26}$

Wenn nun der Insolvenzverwalter ohne Einwilligung oder entgegen dem Willen der jeweiligen Patienten des Vertragsarztes Einblick in diese Unterlagen nimmt, um Abrechnungen erstellen zu können, könnte hierin ein Verstoß des Vertragsarztes gegen die Berufsordnungen für die in Deutschland tätigen Ärztinnen und Ärzte (MBO) zu sehen sein. Nach §9 Abs. 1 S. 1 MBO haben Ärztinnen und Ärzte über das, was ihnen in ihrer Eigenschaft als Ärztin oder Arzt anvertraut oder bekannt geworden ist - auch über den Tod der Patientin oder des Patienten hinaus zu schweigen. ${ }^{27}$ Ein Verstoß kann nach den Heilberufsgesetzen der Länder mit einer Geldbuße bis zu 50000,00 EUR oder mit der Feststellung der Unwürdigkeit zur Ausübung des Berufs geahndet werden. ${ }^{28}$

Darüber hinaus könnte auch ein Verstoß des Arztes gegen $§ 203$ Abs. 1 Nr. 1 StGB angenommen werden. Danach wird mit Freiheitsstrafe bis zu einem Jahr oder mit Geldstrafe bestraft, wer unbefugt ein fremdes Geheimnis, namentlich ein zum persönlichen Lebensbereich gehörendes Geheimnis oder ein Betriebs- oder Geschäftsgeheimnis, offenbart, das ihm als Arzt anvertraut worden oder sonst bekanntgeworden ist. Nicht richtig sein dürfte die insolvenzrechtliche Entscheidung des LG Berlin sein, nach der eine Strafbarkeit des Arztes infolge der Erfüllung der Verpflichtung aus $\S 22$ Abs. 3 Satz 2 InsO („Der Schuldner hat dem vorläufigen Insolvenzverwalter Einsicht in seine Bücher und Geschäftspapiere zu gestatten.“ $)^{29}$ nicht gegeben ist. Denn Tatbestandsvoraussetzung des §203 StGB sei die „unbefugte" Offenbarung eines fremden Geheimnisses. „Unbefugt“ könne aber nicht handeln, wer aufgrund besonderer Gesetze zur Offenbarung berechtigt oder verpflichtet sei. ${ }^{30}$ Soweit das Gesetz - wie $\S 22 \mathrm{Abs} .3$ InsO - einen Anspruch auf Auskunft oder Einsicht geben würde, welcher die Offenbarung eines fremden Geheimnisses einschließt, wäre die Erfüllung dieses Anspruchs durch den Schweigepflichtigen mit Wirkung für das Strafrecht gerechtfertigt. Der Auffassung des LG Berlin ist jedoch wohl nicht zu folgen. Es bestehen insoweit verfassungsrechtliche Be- 
denken, da das allgemeine Persönlichkeitsrecht des einzelnen Patienten nach Art. 1 Abs. 1, Art. 2 Abs. 1 GG im Rahmen einer Abwägung sicherlich schwerer wiegen dürfte, als das letztlich rein finanzielle Interesse der Gläubiger. ${ }^{31}$

Vor diesen Hintergründen ist eine Rechnungslegung durch den Insolvenzverwalter unzulässig. Vielmehr ist die Rechnungslegung in Übereinstimmung mit $\S 1$ Abs. 2 HVM KVNO von dem Vertragsarzt persönlich vorzunehmen.

\section{Gesamterklärung}

Nach §1 Abs. 4 HVM KVNO ist Voraussetzung der Abrechnung, dass die Vertragsärzte die vom Vorstand der KVNO für die Abrechnung festgesetzten Erklärungen auf Vordruck (Gesamtaufstellung) ordnungsgemäß und vollständig abzugeben haben. Dabei sind von den Vertragsärzten die für das jeweilige Quartal von der KVNO zur Verfügung gestellten Vordrucke zu benutzen. In der Gesamtaufstellung ist durch Unterschrift zu bestätigen, dass der Unterzeichner die Verantwortung für die Erfüllung der Abrechnungsvoraussetzungen trägt, weil er sie selbst erfüllt oder sich von deren Erfüllung persönlich überzeugt hat. Eine Veränderung des Erklärungstextes führt zur Unwirksamkeit.

Auch die Abgabe der Gesamterklärung durch den Insolvenzverwalter ist unzulässig. Sie ist in Übereinstimmung mit $\S 1$ Abs. 4 HVM KVNO aus den unter 1 . benannten Gründen ausschließlich von dem Vertragsarzt persönlich abzugeben.

\section{Insolvenzrechtlicher Anspruch gemäß §§97, 98 InsO}

Soweit der insolvente Vertragsarzt sich weigern sollte, die Abrechnung und/oder die Gesamtaufstellung nach $\S 1$ Abs. 1 und 4 HVM KVNO zu erstellen, ergibt sich eine entsprechende Verpflichtung aus $\S \S 97$, 98 InsO, die die Auskunfts- und Mitwirkungspflichten des Vertragsarztes als Schuldner normieren, wobei es vorliegend wohl mehr um die Mitwirkungs- und weniger um die Auskunftspflicht geht. Dabei ist zu berücksichtigen, dass ein insolventer Vertragsarzt an einer Mitwirkung ggf. kein Interesse haben könnte, soweit die sich aus seiner Rechnungslegung bzw. GesamtaufstellungergebendenVergütung an seine Gläubiger weitergeleitet würde.
Nach §97 InsO ist der Vertragsarzt als Schuldner verpflichtet, dem Insolvenzgericht oder dem Insolvenzverwalter über alle das Verfahren betreffenden Verhältnisse Auskunft zu geben. Er hat den Verwalter bei der Erfüllung von dessen Aufgaben zu unterstützen. Der Vertragsarzt ist auch verpflichtet, sich auf Anordnung des Gerichts jederzeit zur Verfügung zu stellen, um seine Auskunfts- und Mitwirkungspflichten zu erfüllen. Er hat alle Handlungen zu unterlassen, die der Erfüllung dieser Pflichten zuwiderlaufen. ${ }^{32}$ §98 InsO listet die Zwangsmittel zur Durchsetzung der Pflichten des Vertragsarztes als Schuldner auf. Danach kann das Gericht den Schuldner zwangsweise vorführen und nach Anhörung und unter Beachtung des Grundsatzes der Verhältnismäßigkeit in Haft nehmen lassen. ${ }^{33}$

Aus den Vorschriften der $\S \S 97,98$ InsO folgt, dass der Vertragsarzt mit den Mitteln der InsO dazu gebracht werden kann, die Abrechnung und / oder die Gesamtaufstellung nach $\S 1$ Abs. 1 und 4 HVM KVNO zu erstellen. Keinesfalls darf dies ersatzweise von dem Insolvenzverwalter durchgeführt werden.

\section{Fristen für die Einreichung von Abrechnungsunterlagen}

Die Abrechnungsfristen sind in $§ 1$ Abs. 5 HVM KVNO geregelt. Danach sind die Abrechnungsunterlagen innerhalb der in Kapitel 3 der seit dem 1.7.2012 gültigen ITRichtlinie der KVNO genannten Abgabetermine einzureichen.

Kapitel 3 der IT-Richtlinie unterscheidet zunächst zwischen 2 Vorlagevarianten: zum einen die Übermittlung der Abrechnung mittels Datenträgern, zum anderen die leitungsgebundene elektronische Übermittlung. Für die Abgabe bzw. Übermittlung der Abrechnung mittels Datenträger gelten die von der jeweils zuständigen Bezirksstelle der KVNO quartalsweise festgelegten Termine. Die leitungsgebundene elektronische Abrechnung des jeweiligen Quartals ist hingegen vom 27. Tag des 3. Quartalsmonats bis zum 12. Tag des ersten Monats im Folgequartal zu übermitteln. Beispiel für das Quartal 2/2012: Die Abrechnung muss zwischen dem 27. Juni 2012 und dem 12. Juli 2012 an die KVNO übermittelt werden. Bei beiden Übermittlungsvarianten sind Übermittlungen außerhalb der Fristen sowie nachträgliche Berichtigungen bzw. Ergänzungen lediglich nach Maßgabe von bzw. mit den Folgen aus $\S 1$ Abs. 5 HVM möglich.

Bei verspäteter Einreichung von Abrechnungsunterlagen finden die Regelungen nach §1 Abs. 5 a) HVM KVNO Anwendung. Eine nachträgliche Berichtigung oder Ergänzung einer unvollständigen Abrechnung für eingereichte Abrechnungsscheine kann der Arzt nach Abgabe der Abrechnungsunterlagen grundsätzlich nicht mehr geltend machen. Ausnahmsweise kann unter der Voraussetzung, dass die nachträgliche Korrektur nicht als geringfügig erscheint, eine Nachbearbeitung nur mit der Folge aus $\S 1$ Abs. 5 b) HVM KVNO verlangt werden. Die Einreichung von Abrechnungsunterlagen nach Ablauf eines Jahres, vom Ende des Kalendervierteljahres an gerechnet, in dem die Leistungen erbracht worden sind, ist ausgeschlossen. Nach $\S 1$ Abs. 5 b) HVM KVNO gilt bei verspäteter Abrechnung folgendes: (1) Vorauszahlungen werden bis zur Vorlage der Abrechnungsunterlagen eingestellt bzw. ermäßigt. (2) Von dem anerkannten Honoraranspruch für die verspätet eingereichte Abrechnung werden $10 \%$, jedoch maximal 10000,00 EUR pauschaliert zur Deckung des zusätzlich entstehenden Aufwandes in Abzug gebracht. Von den vorgenannten Maßnahmen kann auf Antrag ganz oder teilweise abgesehen werden, wenn der Arzt nachweist, dass er die Einreichungsfrist ohne sein Verschulden versäumt hat.

Im Rahmen der Insolvenz eines Vertragsarztes wird man jedoch davon ausgehen müssen, dass diese relativ starren Regelungen nicht zur Anwendung kommen, zumal dies rechtsmissbräuchlich sein dürfte. Hierfür spricht zum einen, dass es sich bei diesen Fristen wohl nicht um Ausschlussfristen handeln dürfte, da für die verspätete Einreichung von Abrechnungsunterlagen ausdrücklich Regelungen aufgestellt worden sind und da auch ansonsten nichts dafür ersichtlich ist, dass die Vertreterversammlung der KVNO, die den HVM erstellt hat, Ausschlussfristen erstellen wollte. ${ }^{34}$ Zum anderen können diese Fristen im Rahmen eines Insolvenzverfahrens nur schwerlich eingehalten werden; dies gilt insbesondere in dem Fall, dass die Abrechnung und / oder die Gesamtaufstellung über die $\S \S 97,98$ InsO erstellt werden müssen. Schließlich ist auch davon auszugehen, dass die Vertreterversammlung der KVNO bei Erstellen des HVM sicherlich nicht eine mögliche Insolvenz von Vertragsärzten bedacht hat. 
Vor diesem Hintergrund wäre es sachgerechter, im Falle der Insolvenz von Vertragsärzten für die Abrechnung die allgemeinen Verjährungsfristen zur Anwendung kommen zu lassen: Mangels spezieller Vorschriften gilt in entsprechender Anwendung des $\S 45$ Abs. 1 SGB I die im Sozialrecht grundsätzlich geltende 4-jährige Verjährungsfrist, sodass die Abrechnung und/oder die Gesamtaufstellung auch noch nach Ablauf der in nach $\S 1$ Abs. 5 HVM KVNO genannten Fristen eingereicht werden können; ${ }^{35}$ Verjährungsbeginn ist danach der Ablauf des Kalenderjahres, an dem der Honoraranspruch des Vertragsarztes gegen die Kassenärztliche Vereinigung entstanden ist.

\section{Zusammenfassung \\ $\nabla$}

Zusammenfassend ist festzustellen, dass Vertragsarztpraxen auch nach Eröffnung des Insolvenzverfahrens, mit grundsätzlich allen Rechten und Pflichten, die sich aus dem Betrieb einer Vertragsarztpraxis ergeben, fortgeführt werden können. Insbesondere haben insolvente Vertragsärzte Vergütungsansprüche gegen die Kassenärztliche Vereinigung. Soweit insolvente Vertragsärzte keine Quartalsabrechnungen und / oder Gesamtaufstellungen erstellen, bestehen die Zwangsmittel der $§ \S 97,98$ InsO (u.a. Verhaftung); keinesfalls dürfen die Insolvenzverwalter diese ersatzweise für die Vertragsärzte erstellen. Die Abrechnungen sind grundsätzlich innerhalb kurzer Fristen bei den Kassenärztlichen Vereinigungen einzureichen; jedoch gilt im Rahmen der Insolvenz von Vertragsärzten eine 4-jährige Verjährungsfrist.

\section{Dr. jur. Michael Ossege, LL.M. Rechtsanwalt und Fachanwalt für Medizinrecht Master of Medical Law}

\section{Rechtsanwälte Wigge \\ Scharnhorststr. 40 \\ 48151 Münster \\ Telefon:0251/53595-0 \\ Telefax: 70251/53595-99 \\ Internet: www.ra-wigge.de \\ E-Mail: kanzlei@ra-wigge.de}

1 Dabei ist zwischen Verbraucher- und Regelinsolvenzverfahren zu unterscheiden. Vorteile des Verbraucherinsolvenzverfahrens: Möglichkeit einer außergerichtlichen Einigung mit den Gläubigern, ggf. unter Androhung ei- ner Regelinsolvenz; Vermeidung stigmatisierender Wirkung eines Insolvenzverfahrens. Vorteile des Regelinsolvenzverfahrens: schnellere Entschuldung des Arztes. Vgl. Christmann, IWW - Praxiswissen für Steuerberater, Praxis Freiberufler-Beratung, Ausgabe $2 / 2012$, S. 45. Im Rahmen dieses Beitrages liegt der Schwerpunkt auf dem Regelinsolvenzverfahren.

2 www.creditreform.de/Deutsch/Creditreform/Aktuelles/Creditreform_News_dyn/Archiv/2006/2006-01-20_Arzt-insolvenzen.jsp (Abrufdatum: 7. 12. 2012).

3 Quelle: BÄK sowie Daten \& Fakten 2011 BZÄK und KZÄBV; laut Spiegel online (www.spiegel. de/wirtschaft /gutverdiener-deutsche-aerzte-gehen-seltener-bankrott-a-614709.html; Abrufdatum 19. 11. 2012) waren im Jahre 2007 genau 223 Arzt- und Zahnarztpraxen zahlungsunfähig, im Jahre 2008 nur noch 165.

4 Quelle: BÄK sowie Daten \& Fakten 2011 BZÄK und KZÄBV.

5 www.destatis.de/DE/Publikationen/StatistischesJahrbuch/StatistischesJahrbuch2011. pdf?_blob=publica-tionFile, Seite 484 (Abrufdatum: 7. 12. 2012).

6 www.ifm-bonn.org/index.php?id=99 (Abrufdatum: 7. 12. 2012) und www.destatis.de/ DE/Publikationen/StatistischesJahrbuch/StatistischesJahrbuch2011.pdf?

blob=publicationFile, Seite 484 (Abrufdatum: 7. 12. 2012).

7 Neuere Zahlen liegen nicht vor (Stand: 19. 11. 2012).

8 Ott/Vuia in Münchener Kommentar zur Insolvenzordnung, Band 1, 2. Aufl. 2007, §80 Rz. 145.

9 Ott/Vuia in Münchener Kommentar zur Insolvenzordnung, Band 1, 2. Aufl. 2007, §80 Rz. 146.

10 Vgl. zur aktuellen Rechtslage u. a. Freudenberg in jurisPK-SGB V, 2. Aufl. 2012, §87b SGB V.

11 http://www.kvno.de/downloads/honorar/ hvm1202_aenderungen.pdf (Abrufdatum: 7. 12. 2012).

12 Unternehmer ist gemäß $\S 2$ Abs. 1 S. 1 UStG, „wer eine gewerbliche oder berufliche Tätigkeit selbständig ausübt." Hiervon wird auch die ärztliche Tätigkeit des Vertragsarztes umfasst, obwohl die entsprechende Leistung im Rahmen des $\S 4$ Nr. 14 UStG nicht umsatzsteuerpflichtig ist. Zu den Hintergründen für die Befreiung vgl. BVerfG, Beschl. v. 29. 10. 19992 BvR 1264/90, jurs = BVerfGE 101, 132 ff. und EuGH, Urt. v. 6. 11. 2003 - C-45/01 (Dornier), Slg. 2003, I-12911-12969 = GesR 2004, 55 (LS); Heintzen / Musil, Das Steuerrecht des Gesundheitswesens, 2. Aufl. 2012, Rz. 359 ff..

13 Vgl. Hesse, Grundzüge des Verfassungsrechts der Bundesrepublik Deutschland, 20. Aufl. 1999, Rz. 72 => praktische Konkordanz.

14 BSG, Urt. v. 17. 6. 2009 - B 6 KA 16/08 R, juris Rz. 37 = SozR 4-2500 §95b Nr. 2; BSG, Urt. v. 20.10.2004 - B 6 KA 67/03 R, juris Rz. 17, GesR 2005, $168=$ SozR 4-2500 $\$ 95$ Nr. 9m.w.N.: Pawlita in jurisPK-SGB V, 2. Aufl. 2012, §95 SGB V Rz. 620.

15 BSG, Urt. v. 24. 11. 1993-6 RKa 70/91, juris Rz. $23=$ SozR $3-2500 \S 95$ Nr. 4.
16 So auch van Zwoll/Mai/Eckardt/Rehborn, Die Arztpraxis in Krise und Insolvenz, 1. Aufl. 2007, S. 106 Rz. 455, 456.

17 Im Ergebnis ebenso Schick, Der Konkurs des Freiberuflers - Berufsrechtliche, konkursrechtliche und steuerrechtliche Aspekte, NJW 1990, S. 2359, 2361.

18 BSG, Urt. v. 10. 5. 2000, Az.: B 6 KA 67/98 B, juris Rz. 20 = NZS 2001,S. 160/161

19 van Zwoll, Ausgewählte Probleme der Arztpraxis in der wirtschaftlichen Krise und Insolvenz, ZMGR 2011, S. 364/364.

20 van Zwoll/Mai/Eckardt/Rehborn, Die Arztpraxis in Krise und Insolvenz, 1. Aufl. 2007, S. 106 Rz. 445 ff.

21 SG Düsseldorf, Urt. v. 25. 05. 2005, S 14 KA 61/04; SG Stuttgart, Urt. v. 12. 12. 2008, Az.: S 10 KA 7601/08 ER; vgl. i. A. Lwowski/Peters, MüKom InsO, §35 Rd. 47

22 http://www.kvno.de/downloads/honorar/ hvm1202_aenderungen.pdf (Abrufdatum: 7. 12. 2012).

23 Mohrbutter in Mohrbutter/Ringstmeier, Handbuch der Insolvenzverwaltung, 8. Aufl. 2007, §6 Rz. 320, 321.

24 Mohrbutter, in Mohrbutter/Ringstmeier, Handbuch der Insolvenzverwaltung, 8. Aufl. 2007, §6 Rz. 321.

25 So Kaiser in Ratzel/Luxenburger, Handbuch Medizinrecht, 2. Aufl. 2011, §13 Rz. 445, 446.

26 Vgl. BVerfG, Entsch. v. 8. 3. 1972-2 BvR 28/71, juris; Giring in: Ratzel/Luxenburger, Handbuch Medizinrecht, 2. Aufl. 2011, §15 Rz. 106; a.A. BGH, Beschl. v. 17. 2. 2005 - IX ZB 62/04, juris Rdnr. 20 ff.; Keller in: Kreft, Insolvenzordnung, 6. Auflage 2011, §36 Rz. 30 Fn. 49.

27 Vgl. §9 Berufsordnung für die nordrheinischen Ärztinnen und Ärzte.

28 Vgl. §60 Heilberufsgesetz NRW.

29 Dies gilt erst recht für den nicht vorläufigen Insolvenzverwalter, vgl. BHG, Urt. v. 15. 3. 2012 - IX ZR 249/09, juris Rz. 16.

30 Vgl. Fischer, Strafgesetzbuch und Nebengesetze, 59. Aufl. 2012, §203 Rz. 31, 37 ff.

$31 \mathrm{Vgl}$. Huber in v. Mangold/Klein/Stark, Kommentar zum Grundgesetz, 6. Aufl. 2010, Art. 19 Rz. 180.

32 Im Falle einer Verbraucherinsolvenz ist die Restschuldbefreiung nach $\S 290$ Abs. 1 Nr. 5 InsO u.U. zu versagen, wenn der Schuldner seinen Verpflichtungen aus $§ 97$ InsO nicht nachkommt.

33 Vgl. Mohrbutter in Mohrbutter/Ringstmeier, Handbuch der Insolvenzverwaltung, 8. Aufl. 2007, §6 Rz. 559, 561; Passauer/Stephan in Münchener Kommentar zur Insolvenzordnung, Band 1, 2. Aufl. 2007, §98 Rz. 15 ff.; Frege/Keller/Riedel, Handbuch der Rechtspraxis, Band 3, Insolvenzrecht, 7. Aufl. 2008, Rz. 849 ff./859.

34 Vgl. jedoch SG Marburg, Urt. v. 26. 9. 2007 - S 12 KA 196/07, juris Rz. 19, wo u. a. darauf hingewiesen wird, dass von Ausschlussfristen erhebliche Wirkungen für die Vergütungsansprüche von Vertragsärzten ausgehen können.

35 Vgl. BSG, Urt. v. 10. 5. 1995-6 RKa 17/94, juris Rz. 14 ff. = SozR 3-1200 §45 Nr. 5 